\title{
Generalized Nörlund summability of fuzzy real numbers
}

\author{
Adem Eroglu, Saban Yilmaz \\ Gaziosmanpaşa University, Faculty of Educational, Department of Mathematics and Vocational High Schools, 60250 Tokat, Turkey
}

Email address:

adem.eroglu@gop.edu.tr (A. Eroglu), saban.yilmaz@gop.edu.tr (S. Yilmaz)

\section{To cite this article:}

Adem Eroglu, Saban Yilmaz. Generalized Nörlund Summability of Fuzzy Real Numbers. Pure and Applied Mathematics Journal. Special Issue: Applications of Geometry. Vol. 4, No. 1-2, 2015, pp. 28-30. doi: 10.11648/j.pamj.s.2015040102.17

\begin{abstract}
Fuzzy set, mathematical modelling in order to some uncertainty in 1965 was described by L. A. Zadeh [7]. In studies on fuzzy sets, fuzzy numbers [5], fuzzy relations [5], fuzzy function [5], fuzzy sequence [4] is defined as concepts. After Nörlund fuzzy and blurry Riez summability have been identified [6]. In this study, fuzzy Generalized Nörlund summability have been defined and Generalized Nörlund summability necessary and sufficient conditions to ensure the regular was investigated.
\end{abstract}

Keywords: Generalized Nörlund Summability, Nörlund Mean Fuzzy, Fuzzy Mean Riesz, Cesaro Mean Fuzzy

\section{Introduction}

This section will be the basic concepts of fuzzy sets.

Definition 1.1. A fuzzy set $\mathrm{A}$ on the universe $\mathrm{X}$ is a set defined by a membership function $\mu_{A}$ representing a mapping

$$
\mu_{A}: X \rightarrow[0,1]
$$

Here the value $\mu_{A}(x)$ for the fuzzy set $A$ is called the membership value or the grade of membership of $x \in X$. The membership value represents the degree of $\mathrm{x}$ belonging to the fuzzy set $A$. [5]

Definition 1.2. Let $D$ denote the set of all closed and bounded intervals $X=\left[a_{1}, a_{2}\right]$ on the real line $R$. For $X, Y \in D$, we define

$$
d(X, Y)=\max \left(\left|a_{1}-b_{1}\right|,\left|a_{2}-b_{2}\right|\right)
$$

where $X=\left[a_{2}, a_{2}\right], Y=\left[b_{2}, b_{2}\right]$. It is known that $(D, d)$ is a complete metric space [6].

Definition 1.3. A fuzzy real number $X$ is called convex if $X(t) \geq X(s) \wedge X(r)=\min (X(s), X(r))$, where $s<t<r$. If there exists $\mathrm{t}_{0} \in \mathrm{IR}$, such that $X\left(t_{0}\right)=1$, then the fuzzy real number $X$ is called normal. A fuzzy real number $X$ is a fuzzy set on $R$ and is a mapping

$$
X: I R \rightarrow I(=[0,1])
$$

associating each real number $t$ with its grade of membership
$X(t)$ [6].

A fuzzy real number $X$ is said to be upper-semicontinuous if for each $\varepsilon>0, X^{-1}([0, a+\varepsilon))$, for all $a \in I$ is open in the usual topology of $R$ [6].

The set of all upper-semicontinuous, normal, convex fuzzy number is denoted by $R(I)$ [6].

Definition 1.4. The $\alpha$-level set of a fuzzy real number $X$, for $0<\alpha \leq 1$ denoted by $X^{\alpha}$ is defined as $X^{\alpha}=\{t \in R: X(t) \geq \alpha\}$; for $\alpha=0$, it is the closure of the strong 0 -cut (that is, the closure of the set $\{t \in I R: X(t)>0\}$ ). Throughout the article $\alpha$ means $\alpha \in(0,1]$ unless otherwise stated [6].

Theorem 1.1. Let $A$ is a fuzzy number if and only if there exists a closed interval $[a, b] \neq \varnothing$ such that

$$
\mu_{A}(x)=\left\{\begin{array}{c}
1, x \in[a, b] \\
l(x), x \in(-\infty, a) \\
r(x), x \in(b, \infty)
\end{array}\right.
$$

where $l$ is a function from $(-\infty, a)$ to $[0,1]$ that is monotonic increasing, continuous from the right, and such that $l(x)=0$ for $x \in\left(-\infty, w_{1}\right) ; r$ is a function from $(b, \infty)$ to $[0,1]$ that is monotonic decreasing, continuous from the left, and such that $r(x)=0$ for $x \in\left(w_{2}, \infty\right)$. [3] 


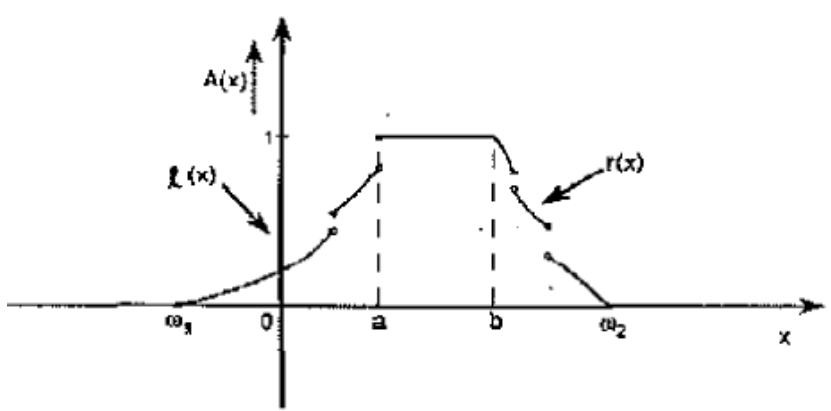

Figure 1.1. Fuzzy number [3]

Definition 1.5. The set $R$ of all real numbers can be embedded in $R(I)$. For each $r \in R, \bar{r} \in R$ is defined by

$$
\bar{r}(t)=\left\{\begin{array}{l}
1, t=r \\
0, t \neq r
\end{array} \quad[6] .\right.
$$

Definition 1.6. Let $\bar{d}: R(I) X R(I) \rightarrow I R$ be defined by

$$
\bar{d}(X, Y)=\sup _{0 \leq \alpha \leq 1} d\left(X^{\alpha}, Y^{\alpha}\right) .
$$

Then $\bar{d}$ defines a metric on $R(I)$. It is well known that $(R(I), \bar{d})$ is a complete metric space. The additive identity and multiplicative identity in $R(I)$ are denoted by $\overline{0}$ and $\overline{1}$ respectively [6].

\section{Preliminaries}

Fuzzy sequence defined on fuzzy sets in this section will be Reisz and Nörlund averages. A fuzzy set of samples to be explained.

Definition 2.1. A sequence $\bar{A}=\left(\bar{A}_{k}\right)$ of fuzzy numbers is said to be convergent to the fuzzy number $\bar{A}_{0}$, written as $\lim _{k \rightarrow \infty} \bar{A}_{k}=\bar{A}_{0}$, if for every $\varepsilon>0$ there exists a positive integer $N$ such that $d\left(\bar{A}_{k}, \bar{A}_{0}\right)<\varepsilon$ for every $k>N$. [1]

Example 2.1.

$$
\bar{A}_{k}(x)=\left\{\begin{array}{cc}
\frac{k}{k+2} x+\frac{2-3 k}{k+2} & , x \in\left[\frac{3 k-2}{k}, 4\right] \\
\frac{-k}{k+2} x+\frac{5 k+2}{k+2} & , x \in\left[4, \frac{5 k+2}{k}\right] \\
0 & , \quad x \notin\left[\frac{3 k-2}{k}, \frac{5 k+2}{k}\right]
\end{array}\right.
$$

from of $\bar{A}=\left(\bar{A}_{k}\right)$ Consider the fuzzy number sequence. Limit of this sequence,

$$
\bar{A}_{0}(x)=\left\{\begin{array}{ccc}
x-3 & , & x \in[3,4] \\
-x+5 & , & x \in[4,5] . \\
0 & , & x \notin[3,5]
\end{array}\right.
$$

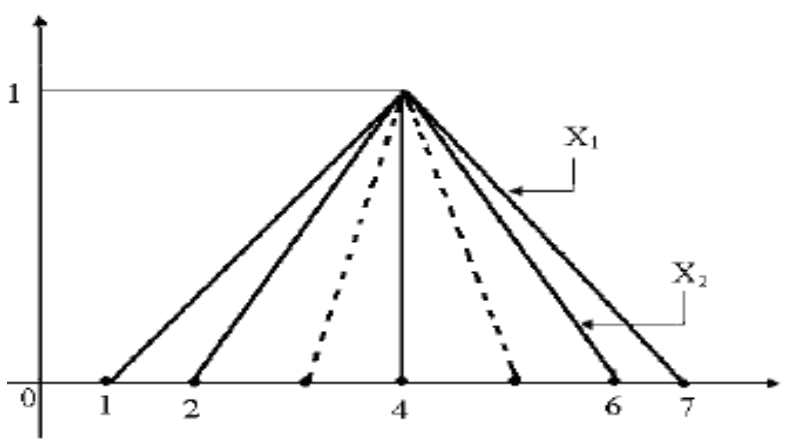

Figure 1.2. $\left(\bar{A}_{k}\right)$ Fuzzy number sequence $\bar{A}_{0}$ the convergence of the fuzzy number [2]

Definition 2.2. Let $\left(p_{n}\right)$ be a sequence of non-negative real numbers which are not all zero and

$$
P_{n}=p_{1}+p_{2}+\ldots+p_{n}
$$

for all $n \in N$. A sequence $\left(\bar{s}_{n}\right)$ of fuzzy real numbers is said to be summable by Nörlund mean $(\bar{N}, p)$ to $\bar{L}$, if

$$
\bar{d}\left(\frac{1}{P_{n}} \sum_{v=1}^{n} p_{n-v+1} \bar{s}_{v}, \bar{L}\right) \rightarrow 0
$$

as $n \rightarrow \infty$.

Definition 2.2. Let $\left(p_{n}\right)$ be a sequence of non-negative real numbers which are not all zero and

$$
P_{n}=p_{1}+p_{2}+\ldots+p_{n}
$$

for all $n \in N$. A sequence $\left(\bar{s}_{n}\right)$ of fuzzy real numbers is said to be summable by Riesz mean $(\bar{R}, p)$ to $\bar{L}$, if

$$
\bar{d}\left(\frac{1}{P_{n}} \sum_{v=1}^{n} p_{v} \bar{s}_{v}, \bar{L}\right) \rightarrow 0
$$

as $n \rightarrow \infty$.

\section{Generalized Nörlund Summability of Fuzzy Real Numbers}

This section will be defined the fuzzy generalized Nörlund summability and explored regularity conditions on the summability.

Definition 3.1. Let $\left(p_{n}\right),\left(q_{n}\right)$ be a sequence of non-negative real numbers which are not all zero and

$$
r_{n}=\sum_{v=0}^{n} p_{n-v} q_{v}
$$

for all $n \in N$. A sequence $\left(\bar{s}_{n}\right)$ of fuzzy real numbers is said to be summable by Nörlund summability $(\bar{N}, p, q)$ to $\bar{L}$, if 


$$
\bar{d}\left(\frac{1}{r_{n}} \sum_{v=1}^{n} p_{n-v+1} q_{v} \bar{s}_{v}, \bar{L}\right) \rightarrow 0
$$

as $n \rightarrow \infty$.

Theorem 3.1. Let $\left(p_{n}\right),\left(q_{n}\right),\left(r_{n}\right)$ be sequences defined in the defenition 2.1. Then, $(\bar{N}, p, q)$ regular if and only if $\lim _{n \rightarrow \infty} \frac{p_{n}}{r_{n}}=0$.

Proof: Sufficiency: Let $\left(\bar{s}_{n}\right)$ be any any convergent sequence of fuzzy real numbers, and $\lim _{n \rightarrow \infty} \bar{s}_{n}=\bar{L}$. Without loss of generality, we may assume that $\bar{L}=\overline{0}$. For a fixed $\varepsilon>0$ there exists no such that, $\bar{d}\left(s_{n}, \overline{0}\right)<H$ for $n \geq n_{0}$. If $\lim _{n \rightarrow \infty} \frac{p_{n}}{r_{n}}=0$ then for all $\varepsilon>0$ there exists $n_{1} \in I N$ such that,

$$
\frac{p_{n}}{r_{n}}<\frac{\varepsilon}{2 H \max \left(n_{0}, n_{1}\right)}
$$

for $n>n_{1}$. Let $n_{2}=\max \left(n_{0}, n_{1}\right)$ and assume $\bar{d}\left(\bar{s}_{n}, \overline{0}\right)<\frac{\varepsilon}{2}$ for all $n>n_{2}$. Then,

$$
\frac{p_{n}}{r_{n}}<\frac{\varepsilon}{2 H \max \left(n_{0}, n_{1}\right)} .
$$

So for all $n>n_{2}$ we obtain.

$$
\begin{aligned}
& \bar{d}\left(\frac{1}{r_{n}} \sum_{v=1}^{n} p_{n-v+1} q_{v} \bar{s}_{v}, \overline{0}\right) \\
& \leq \bar{d}\left(\frac{1}{r_{n}} \sum_{v=1}^{n_{2}} p_{n-v+1} q_{v} \bar{s}_{v}, \overline{0}\right)+\bar{d}\left(\frac{1}{r_{n}} \sum_{v=n_{2}+1}^{n} p_{n-v+1} q_{v} \bar{s}_{v}, \overline{0}\right) \\
& =\bar{d}\left(\frac{1}{r_{n}}\left(p_{n} q_{1} \bar{s}_{1}+\ldots+p_{n-n_{2}+1} q_{n_{2}} \bar{s}_{n_{2}}\right), \overline{0}\right)+ \\
& \quad+d\left(\frac{1}{r_{n}}\left(p_{n-n_{2}} q_{n_{2}+1} \bar{s}_{n_{2}+1}+\ldots+p_{1} q_{n} \bar{s}_{n}\right), \overline{0}\right) \\
& =\frac{p_{n} q_{1}}{r_{n}} \bar{d}\left(\bar{s}_{1}, \overline{0}\right)+\ldots+\frac{p_{n-n_{2}+1} q_{n_{2}}}{r_{n}} \bar{d}\left(\bar{s}_{n_{2}}, \overline{0}\right)+ \\
& \quad+\frac{p_{n-n_{2}} q_{n_{2}+1}}{r_{n}} \bar{d}\left(\bar{s}_{n_{2}+1}, \overline{0}\right)+\ldots+\frac{p_{1} q_{n}}{r_{n}} \bar{d}\left(\bar{s}_{n}, \overline{0}\right) \\
& \leq \frac{p_{n} q_{1}}{r_{n}} H+\ldots+\frac{p_{n-n_{2}+1} q_{n_{2}}}{r_{n}} H+\frac{p_{n-n_{2}} q_{n_{2}+1}}{r_{n}} \cdot \frac{\varepsilon}{2}+\ldots+\frac{p_{1} q_{n}}{r_{n}} \frac{\varepsilon}{2}
\end{aligned}
$$

$\leq \frac{\varepsilon}{2 H n_{2}} H+\ldots+\frac{\varepsilon}{2 H n_{2}} H+\left(\frac{p_{n-n_{2}}}{r_{n}}+\ldots+\frac{p_{1}}{r_{n}}\right) \frac{\varepsilon}{2}$

$<\frac{\varepsilon}{2}+\frac{\varepsilon}{2}=\varepsilon$

Necessity: Let $(\bar{N}, p, q)$ to get regular and $\bar{e}_{1}=(\overline{1}, \overline{0}, \overline{0}, \ldots)=\left(\bar{s}_{v}\right)$ consider the sequence. Then,

$$
\lim _{v \rightarrow \infty} \bar{s}_{v}=\overline{0}
$$

and so

$$
\lim _{n \rightarrow \infty} \frac{p_{n}}{r_{n}}=0
$$

since

$$
\lim _{n \rightarrow \infty} \bar{d}\left(\sum_{v=1}^{n} \frac{p_{n-v+1} q_{v}}{r_{n}} \bar{e}_{1}, \overline{0}\right)
$$

\section{Results}

In this section, fuzzy generalized summability Nörlund been identified and will be investigated regularity conditions on the summability.

$(\bar{N}, p, q)$ regular if and only if $\lim _{n \rightarrow \infty} \frac{p_{n}}{r_{n}}=0$ we obtained.

\section{References}

[1] Aytar, S., 2003, Statistical limit points of sequences of fuzzy numbers, Elsevier, Information Sciences 165 (2004) 129-138.

[2] Çınar, M. 2007, Bulanık Sayı Dizileri ve İstatistiksel Yakınsaklık, Fırat Üniversitesi, Fen Bilimleri Enstitüsü, Yüksek Lisans Tezi.

[3] George, J. K., and Bo Yuan, Fuzzy Sets and Fuzzy Logic, Theory and Applications, USA, 1995.

[4] Moore, R. E., 1979, Methods and Apllications of Interval Analysis, SIAM Philadelphia.

[5] Tanaka, K., 1991. An Introduction to Fuzzy Logic for Practical Applications, Kanazawa, Japan.

[6] Tripathy, B. C., Baruah, A., Nörlund and Riesz mean of sequences of fuzzy real numbers, Applied Mathematics Letters 23 (2010) 651-655.

[7] Zadeh, L.A. (1973) Outline of a new approach to the analysis of complex systems and decision processes. IEEE Trans. Man Cybernetics 3: 28-44. 